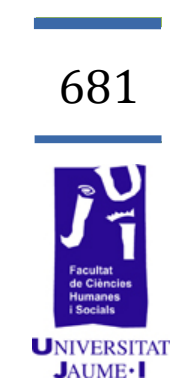

\title{
El color rojo en español. Un estudio de su connotación basado en corpus
}

Alejandro Teruel Vidal al096312@uji.es 
Las expresiones lingüísticas con sentido figurado se encuentran en el lenguaje cotidiano de todos los hablantes $y$, a menudo, sirven para explicar conceptos abstractos a través de otros más concretos. Nuestro objetivo es demostrar que algo tan usual como un término cromático dentro de una expresión de este tipo puede ser el vehículo perfecto para hacer entender ideas abstractas con sentido metafórico o metonímico. Para ello usaremos una herramienta clave en el análisis lingüístico: el corpus. Mediante los corpus realizaremos un pequeño estudio descriptivo que nos permita ver de qué manera los términos del color favorecen una connotación determinada.

Palabras clave: corpus lingüísticos, corpus electrónicos, estudios de traducción basados en corpus, colores, rojo, metáfora, metonimia, prosodia semántica.

\section{Introducción: investigación en Traducción con corpus textuales}

Si bien la traducción es una actividad que se lleva a cabo desde hace milenios, no fue hasta la segunda mitad del siglo xx cuando se empieza a sentir la necesidad de profundizar en el concepto de Traducción como disciplina. El gran número de avances que se produjeron durante la primera mitad del siglo $\mathrm{xx}$, como el desarrollo de la tecnología y el aumento de las relaciones internacionales, marcaron un punto de inflexión en el campo de la Traducción, que experimentó un boom debido a la aparición de nuevas formas de traducción e interpretación que marcarían un antes y un después en la manera de ver la disciplina.

Este desarrollo de la tecnología facilitó la aparición de herramientas que permitieran profundizar en el estudio de la Traducción y del proceso traductológico, como la herramienta que ocupa nuestro estudio: el corpus lingüístico. Si hablamos de corpus, es imprescindible mencionar el trabajo de Mona Baker (1993), precursora de los estudios de traducción basados en corpus. Las investigaciones de Baker ofrecen un punto de vista empírico que demuestra la gran utilidad de los corpus para poder confirmar hipótesis extraídas mediante este tipo de catálogos textuales. Los corpus se presentan como una de las principales herramientas metodológicas para el estudio de la traducción. Un corpus lingüístico es un conjunto de datos o textos que puede servir de base a una investigación y podemos encontrar todo tipo de corpus lingüísticos, como los monolingües, bilingües, paralelos, comparables, alineados, etc. Las características de nuestro estudio harán que nos centremos en los corpus monolingües, ya que no vamos a realizar por el momento un estudio comparativo y queremos centrarnos en cómo funciona la lengua española en este ámbito de estudio. 
Algunos de los factores que tendremos en cuenta a la hora de seleccionar el corpus que vamos a utilizar para nuestra investigación es la posibilidad de restringir las búsquedas en el corpus, la tipología textual que encontramos en él $y$, sobre todo, el tamaño que tiene el corpus para poder trabajar con una mayor cantidad de datos y que los resultados tengan una mayor envergadura o una mayor validez. Trabajar con un corpus de mayor tamaño puede suponer un problema, ya que el volumen de datos que se obtendrá durante su extracción será también mayor y esto hará que sea algo más difícil comprobar las teorías que tenemos y realizar generalizaciones. Sin embargo, consideramos que si durante el proceso de extracción hemos analizado un mayor número de datos, la validez de los resultados obtenidos será mayor, por lo que es importante ver cómo trabajamos con estos datos para poder llegar a una generalización válida.

El corpus nos permitirá obtener muestras reales de expresiones con sentido figurado, por lo que antes de empezar con el estudio es importante resaltar tres aspectos clave que analizaremos de forma individual: la metáfora, la metonimia y la prosodia semántica. Por lo que respecta a la metonimia, Lakoff y Johnson (1980: 39) la definieron como una manera de «conceptualizar una entidad mediante su relación con otra». Consiste en comprender un concepto abstracto utilizando otro que es más fácil de entender porque nos es más conocido y que guarda algún tipo de relación semántica con el primer término más abstracto. Un ejemplo es la frase "La Casa Blanca no se ha pronunciado al respecto» donde "Casa Blanca» hace referencia a "los portavoces de la Casa Blanca». Los mismos autores estudiaron profundamente la metáfora conceptual, que sirve para explicar un concepto por medio de otra realidad con la que guarda cierta relación de semejanza. Ejemplo de ello sería la metáfora conceptual «El amor es un viaje», algo que se explica mediante frases como «Hemos llegado a una encrucijada en nuestra relación» o "Tenemos que ir un poco más despacio». Lakoff y Johnson revolucionaron la lingüística cognitiva gracias a sus estudios en metáfora y metonimia conceptual, porque se considera que mediante estos dos mecanismos tan simples y que se encuentran tan a la orden del día, es posible entender a menudo cómo se estructuran diversos aspectos del pensamiento humano.

Ya que vamos a analizar expresiones con sentido figurado y a extraer conclusiones sobre cuál es la motivación para utilizar el color rojo en algunas expresiones concretas, es importante considerar un aspecto de la connotación, que es el concepto de prosodia semántica, ya estudiado en el año 1993 por Louw. Por un lado, tenemos la connotación de una palabra, que es fácil de identificar solo con un conocimiento enciclopédico general. Este sería el caso de la palabra «muerte», que ya tiene para el hablante una connotación negativa solo con saberse qué es y en qué contextos aparece. Por otro lado, la prosodia semántica se presenta, en cambio, en una palabra neutra cuando esta se combina con otra palabra que le confiere este valor semántico, ya sea positivo o 
negativo. Esto ocurre cuando usamos palabras como, por ejemplo, "causar» o "suceder», que por sí solas no tienen ningún tipo de connotación, pero que en numerosas ocasiones asociamos a cosas negativas con otras palabras que le confieren este valor («causar daño» o "suceder una tragedia»). Estas coocurrencias o colocaciones pueden ser muy interesantes para estudiar el lenguaje figurado presente en algunas expresiones, por lo que utilizar un corpus textual permite analizar de forma contextualizada aquellas que presentan rasgos de prosodia semántica y sus combinaciones posibles.

\section{Objetivos}

El objetivo de este estudio introductorio es averiguar cuál es el papel de los términos cromáticos cuando se trata de favorecer una connotación determinada en las expresiones en sentido figurado. Sabemos que los términos que hacen referencia a los colores están presentes en un gran número de expresiones figuradas, como "ponerse rojo como un tomate» o "estar rojo de ira», por lo que nuestro fin es saber en qué metáforas o metonimias conceptuales interviene el color rojo y en qué grado este color determina la expresión figurada que hemos analizado.

\section{Metodología}

Teniendo en cuenta los dos planteamientos que presentó la académica Tognini-Bonelli (2001), nuestro estudio ofrecerá un enfoque guiado por corpus. La autora diferenció entre el corpus-based approach (enfoque basado en corpus) y el corpus-driven approach (enfoque guiado por corpus). Mientras que el enfoque basado en corpus es un planteamiento deductivo por el que se utiliza un corpus para comprobar teorías que ya se tenían planteadas mediante ejemplos ilustrativos, el enfoque guiado por corpus ofrece un planteamiento inductivo donde se utiliza un corpus para extraer datos que permitirán hacer generalizaciones sobre aspectos, conceptos o teorías que son nuevos o todavía desconocidos para el investigador. Ya que no tenemos una hipótesis preestablecida sobre qué puede motivar el uso de los términos cromáticos en el lenguaje figurado, utilizaremos el segundo planteamiento durante nuestra investigación y a través del uso del corpus extraeremos las diversas conclusiones. A continuación pasaremos a describir de forma concreta cuál va a ser nuestra metodología y en qué fases dividiremos nuestra investigación.

\subsection{Selección del corpus monolingüe}

Es importante, primero que nada, diferenciar algunos conceptos de terminología básica de corpus. Cada corpus textual se compone de un número determinado de palabras (en inglés, tokens) que son cada unidad 
lingüística que aparece en el corpus y dentro de este conjunto de palabras hay un número determinado de formas (en inglés, types), que son aquellas palabras distintas dentro del corpus. Una vez definidos estos conceptos podemos comentar que, de forma previa a la realización de nuestro estudio, hemos llevado a cabo un análisis de una serie de corpus lingüísticos, entre los que se encuentran, por ejemplo, los diversos del Banco de Datos de la Real Academia Española (Corpus de Referencia del Español Actual, Corpus Diacrónico del Español, Corpus nuclear del Nuevo Diccionario Histórico del Español, Corpus del Español del Siglo XXI) o el Corpus del Español de Mark Davies. Tras valorar cuál se iba a adaptar más a nuestras necesidades decidimos decantarnos por el de Mark Davies, que contiene alrededor de 100 millones de palabras y se compone de textos que abarcan el período que va del siglo XIII al Xx.

El Corpus del Español de Mark Davies ( $\mathrm{CdE}$, de ahora en adelante) es un corpus anotado que da muchas posibilidades, ya que permite restringir la búsqueda de forma cómoda y organizar los resultados de manera que sea muy fácil extraer conclusiones de forma rápida y organizada. Por todas las opciones de trabajo que ofrece y por el carácter de nuestra investigación, consideramos que es el corpus que más se adapta a nuestro estudio y es el que hemos elegido para realizar el análisis de las expresiones con el término cromático «rojo».

\subsection{Proceso de búsqueda en el corpus seleccionado}

Llevaremos a cabo un análisis cuantitativo y cualitativo de los distintos datos del corpus. El análisis cuantitativo servirá para ver, de forma general, cuántas expresiones con el término cromático «rojo» ofrecen carácter fraseológico y el análisis cualitativo consistirá en ver, dentro de las expresiones con sentido figurado, de qué tipo de fraseologismo se trata, cuál es la connotación encontrada y cuáles son las coocurrencias que presenta a menudo. Para terminar, nos gustaría realizar una clasificación no exhaustiva de las diferentes connotaciones encontradas en las expresiones con color rojo.

Hemos valorado cuál va a ser nuestra forma de búsqueda y, en primer lugar, habíamos pensado realizar las búsquedas usando el asterisco $(*)$, roj*, para que además de obtener el término «rojo», obtuviéramos «roja», "rojos», «rojas», sin embargo, una búsqueda así nos ofrece 52 resultados, entre los que se encuentran palabras derivadas como "rojinegro», "rojizo» o "rojillo», por lo que la búsqueda por lemas será la forma que más se adaptará a nuestros objetivos. El CdE utiliza los corchetes en este tipo de búsquedas, y si realizamos una búsqueda tentativo de [rojo] obtendremos los siguientes resultados: 


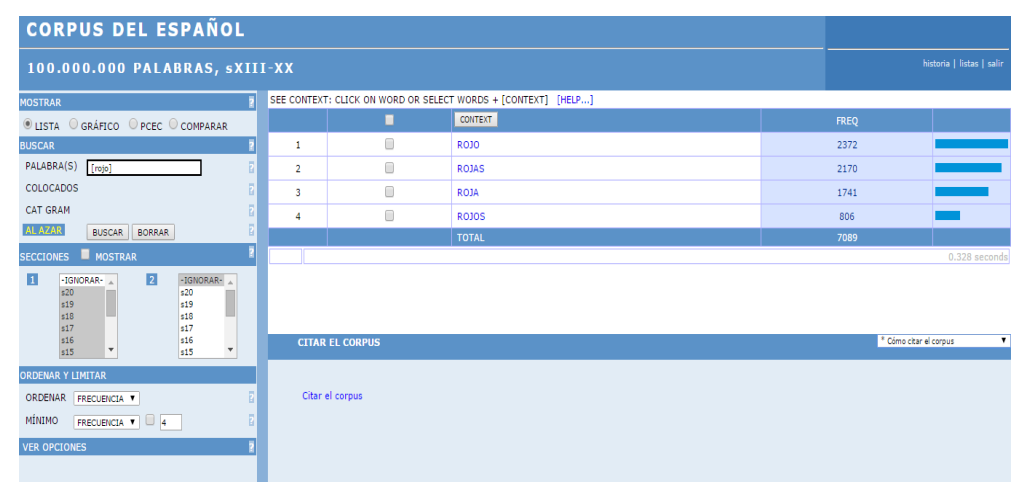

Figura 1

La búsqueda por lemas nos ofrece los términos "rojo», "roja», «rojos» y «rojas» y, como podemos ver en la figura anterior, el CdE ofrece otras posibilidades muy interesantes para ampliar o restringir las búsquedas. Es posible buscar colocaciones con una palabra determinada o también buscar colocaciones con palabras que tengan una categoría gramatical concreta. La búsqueda de colocaciones sería interesante en la fase posterior, cuando procedamos a analizar expresiones metafóricas concretas y los rastros de prosodia semántica de diversas unidades léxicas.

Por último, queremos resaltar dos aspectos de la búsqueda en el CdE. Por un lado, el corpus realiza de forma predefinida la búsqueda de textos solo de los siglos XX y XIX, pero para obtener más resultados y que la variedad de textos sea mayor, hemos decidido realizar una búsqueda del término "rojo» en todos los siglos que están representados en el CdE (del siglo XIII al siglo XX). Por otro lado, el CdE permite realizar la búsqueda en cuatro secciones diferentes: la sección «Oral» presenta textos orales, "Fic» recoge textos de ficción, "Per» contiene textos de periódicos y revistas $y$, por último, "Acad» ofrece textos académicos. Hemos seleccionado todas las secciones del corpus porque puede ser interesante ver en qué contextos aparecen determinadas expresiones metafóricas y también porque la sección de textos orales puede ayudarnos a demostrar que este tipo de expresiones en sentido figurado están a la orden del día en el habla de los españoles y no son solo una herramienta embellecedora de la lengua escrita.

\subsection{Proceso de clasificación de los resultados obtenidos}

El corpus ordena automáticamente los diferentes resultados por sección, siglo y texto y nuestro objetivo es obtener resultados que representen todas las secciones del corpus y no solo los que primero aparezcan. Es aquí donde hemos dejado de lado la forma de búsqueda por listas que ofrece el $\mathrm{CdE}$ de forma predefinida y hemos pasado a realizar la búsqueda mediante $\mathrm{KWIC}$, que responde a las siglas en inglés de Keyword in Context o lo que es lo mismo en español, palabra clave en su contexto (PCEC). Se trata de la expresión de una palabra clave (en nuestro caso «rojo») y el contexto que aparece delante y detrás de la 
palabra para que podamos comprobar el significado de forma clara. En el Corpus del Español de Mark Davies se presenta de la siguiente forma:

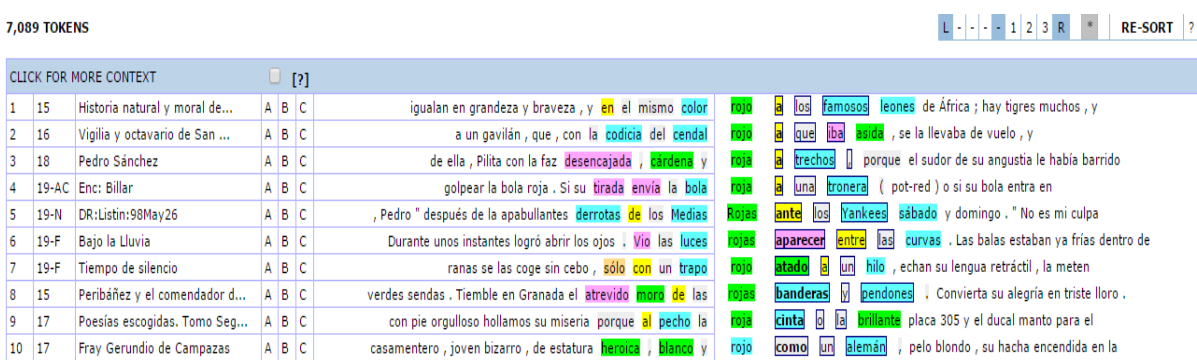

Figura 2

Al ser un estudio introductorio, realizaremos la clasificación de forma casi manual, siguiendo el método Pragglejaz, que se explica en la siguiente fase. Antes que nada, queremos explicar los colores representados en la figura anterior. Cada color hace referencia a una categoría gramatical concreta:

Figura 3

Esto es posible porque el CdE es un corpus anotado, sin embargo, la anotación de un corpus se hace de forma semiautomática y a veces puede fallar, por lo que hay que estar seguro antes de extraer conclusiones. Ejemplo de ello es el KWIC 1 de la Figura 2, donde "famosos» está anotado como un sustantivo, ya que aparece en azul, al igual que "leones», pero en este caso "famosos» cumple la función de adjetivo por lo que debería aparecer subrayado en color verde. Esta opción puede ser muy práctica, pero se deberá usar con sumo cuidado y de forma orientativa para no presentar datos erróneos durante el estudio.

A la hora de determinar qué expresiones tienen sentido figurado hemos decidido seguir el método Pragglejaz, que pretende buscar el significado metafórico de las palabras. Uno de sus precursores en la escuela holandesa, Steen, propuso en su artículo de 2007 los siguientes pasos para dar con la metáfora canónica en el discurso:

- Leer el texto completo para entender de qué se trata.

- Decidir cuáles son los límites de las palabras.

- Establecer el significado contextual de las palabras examinadas.

- Determinar el significado básico de la palabra (más concreta, orientada a las personas y específica).

- Decidir si el significado básico de la palabra es lo suficientemente distinto del significado contextual.

- Decidir si el significado contextual de la palabra se puede relacionar con el significado más básico mediante algún tipo de similitud. 
Hemos decidido dividir el proceso de clasificación de los resultados del $\mathrm{CdE}$ en dos fases. En una primera fase llevaremos a cabo una selección de 700 palabras en su contexto, algo que nos permitirá abordar un estudio más cuantitativo antes de entrar en materia y nos permitirá tener una muestra de expresiones que analizaremos posteriormente. En la segunda fase se procederá a analizar unas 100 coocurrencias para así poder clasificar de forma más sencilla el papel de los términos del color dentro de estas coocurrencias.

\subsubsection{Clasificación de las expresiones metafóricas}

En un primer acercamiento realizamos una búsqueda de 200 expresiones u oraciones en las que aparecía el término "rojo» y en una segunda parte de la primera fase decidimos ampliar la búsqueda a 500 expresiones más ya que comprobamos que con 200 expresiones solamente no teníamos suficiente para extraer la información que necesitábamos para generalizar, por lo que en total analizamos $700 \mathrm{KWIC}$ con el término «rojo». A continuación presentamos las expresiones que hemos considerado que tienen sentido metafórico, según el método Pragglejaz:

\begin{tabular}{|c|c|c|c|c|c|}
\hline 1 & $19-\mathrm{F}$ & $\begin{array}{l}\text { La víspera y } \\
\text { el día }\end{array}$ & $\begin{array}{c}\text { sus citas amorosas en } \\
\text { ese mismo momento. } \\
\text { El coronel, }\end{array}$ & rojo & $\begin{array}{l}\text { de ira pidió a su leal } \\
\text { informante que lo } \\
\text { acompañara como }\end{array}$ \\
\hline 2 & $19-A C$ & $\begin{array}{l}\text { Enc: China } \\
\text { (república) }\end{array}$ & $\begin{array}{c}\text { sobre las relaciones } \\
\text { exteriores. La actitud } \\
\text { de los } \\
\text { guardias }\end{array}$ & rojos & \begin{tabular}{|l|} 
inspiró \\
comportamientos \\
similares en Hong \\
Kong que dieron \\
\end{tabular} \\
\hline 3 & $19-N$ & $\begin{array}{l}\text { Cuba: } \\
\text { CubaNet: } \\
\text { 98May5 }\end{array}$ & $\begin{array}{c}\text { hemos decidido hacer } \\
\text { esta ponencia. El } \\
\text { problema de la } \\
\text { carne }\end{array}$ & roja & $\begin{array}{c}\text { tiene solución } \\
\text { solamente dando } \\
\text { autorización a todo el } \\
\text { que }\end{array}$ \\
\hline 4 & $19-A C$ & $\begin{array}{c}\text { Enc: Teoría } \\
\text { cuántica }\end{array}$ & $\begin{array}{l}\text { sin reflejar ninguna). } \\
\text { Un cuerpo a } \\
\text { temperatura alta - al }\end{array}$ & rojo & $\begin{array}{l}\text { vivo- emite la mayor } \\
\text { parte de su radiación } \\
\text { en las zonas de }\end{array}$ \\
\hline 5 & $19-N$ & $\begin{array}{l}\text { Arg: Prensa: } \\
\text { 260_COY }\end{array}$ & $\begin{array}{l}\text { navegación a las } 8,20 \\
\text { se recibió a bordo un } \\
\text { alerta }\end{array}$ & rojo & $\begin{array}{c}\text { E El ataque del } \\
\text { guardacostas por parte } \\
\text { de aviones británicos } \\
\text { fue }\end{array}$ \\
\hline 6 & $19-\mathrm{F}$ & $\begin{array}{l}\text { La catedral } \\
\text { sumergida }\end{array}$ & $\begin{array}{c}\text { de gris, que ladean mis } \\
\text { mejillas. Las sentí } \\
\text { volverse }\end{array}$ & rojas & $\begin{array}{l}\text { causa de la ira que } \\
\text { se adueñó de mí al } \\
\text { proseguir }\end{array}$ \\
\hline 7 & $19-N$ & $\begin{array}{l}\text { Méx: } \\
\text { Yucatán: } \\
\text { 97Jun13 }\end{array}$ & $\begin{array}{c}\text { m. } 46 \text { Stoitchkov, } \\
\text { m. } 72 \text { Ivanov: . m. 87, } \\
\text { Tarjeta }\end{array}$ & roja & $\begin{array}{l}\text { a Nankov por doble } \\
\text { amonestación. } \\
\text { Incidencias: Primer } \\
\text { partido }\end{array}$ \\
\hline 8 & 18 & $\begin{array}{l}\text { Las ilusiones } \\
\text { del doctor } \\
\text { Faustino }\end{array}$ & $\begin{array}{c}\text { qué desenlace van a } \\
\text { tener? D. Faustino se } \\
\text { puso }\end{array}$ & rojo & $\begin{array}{c}\text { como la grana y bajó } \\
\text { los ojos al suelo, } \\
\text { guardando silencio }\end{array}$ \\
\hline 9 & 18 & $\begin{array}{l}\text { El doncel de } \\
\text { Don Enrique }\end{array}$ & $\begin{array}{c}\text { de los feudales señores } \\
\text { de la época. El sol, }\end{array}$ & rojo & $\begin{array}{l}\text { como la lumbre, } \\
\text { despidiendo sus rayos }\end{array}$ \\
\hline
\end{tabular}




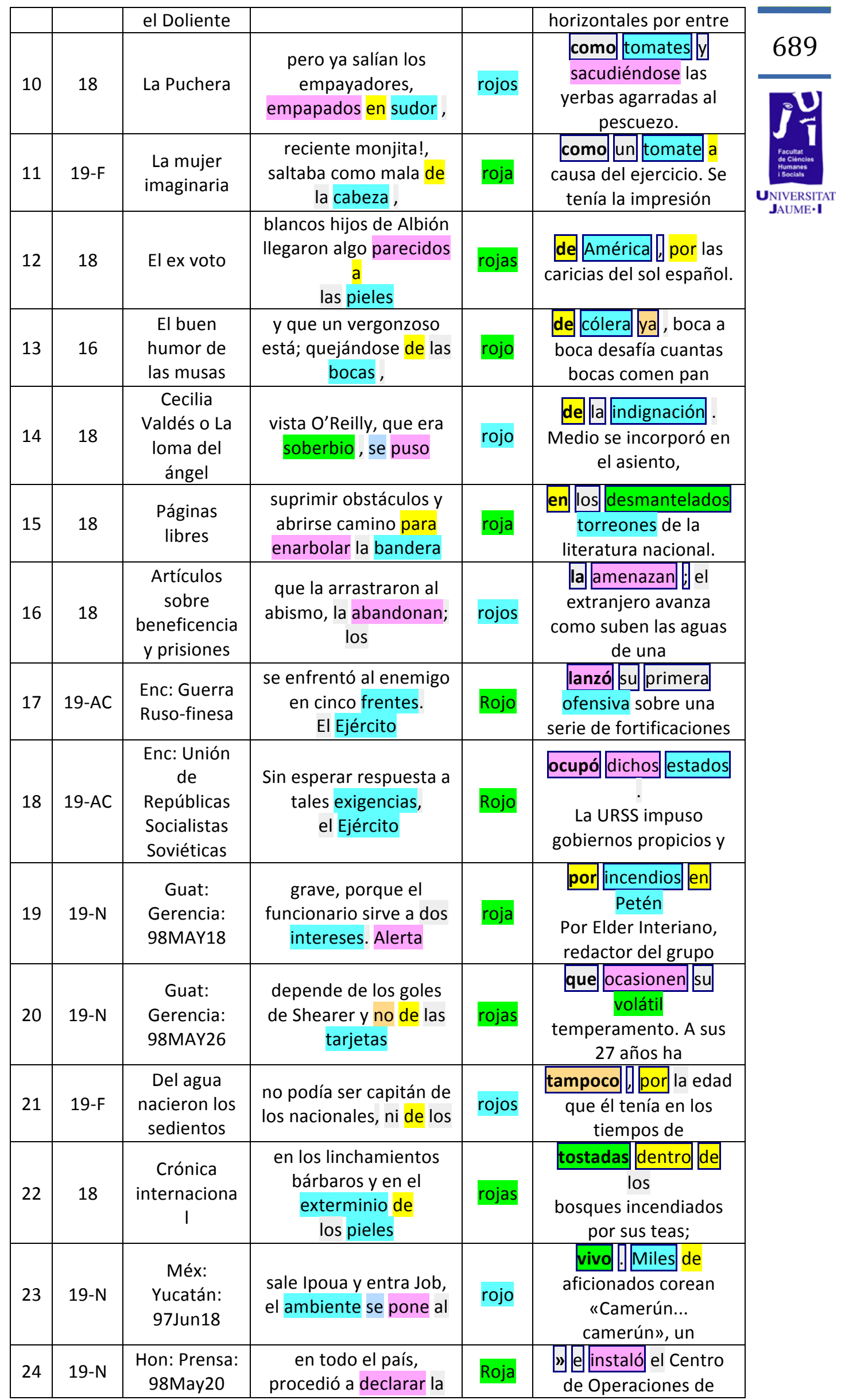




\begin{tabular}{|c|c|c|c|c|c|}
\hline & & & «Alerta & & Emergencia, en la \\
\hline 25 & $19-N$ & $\begin{array}{l}\text { Cuba: } \\
\text { CubaNet: } \\
\text { 98Mar10 }\end{array}$ & $\begin{array}{c}\text { de la isla continúa sin } \\
\text { comer carne blanca, } \\
\text { pues la }\end{array}$ & roja & $\begin{array}{c}\text { como se diría en } \\
\text { portugués al decir del } \\
\text { cubano, }\end{array}$ \\
\hline 26 & 18 & Morsamor & $\begin{array}{c}\text { la raza negra, la } \\
\text { amarilla y la raza de } \\
\text { piel }\end{array}$ & roja & $\begin{array}{l}\text { cuyos individuos se } \\
\text { esparon atlantes y se } \\
\text { esparon por el }\end{array}$ \\
\hline 27 & $19-\mathrm{N}$ & $\begin{array}{l}\text { DR: Listin: } \\
\text { 98May30 }\end{array}$ & $\begin{array}{c}\text { una situación en la que } \\
\text { José Adames } \\
\text { fue penalizado } \\
\text { con tarjeta }\end{array}$ & roja & $\begin{array}{l}\text { hecho que provocó } \\
\text { posteriormente la } \\
\text { salida del dirigente }\end{array}$ \\
\hline 28 & $\begin{array}{l}19- \\
\text { OR }\end{array}$ & $\begin{array}{l}\text { Habla Culta: } \\
\text { Santiago: } \\
\text { M10 }\end{array}$ & $\begin{array}{l}\text { guerra, la vio tan cruel, } \\
\text { o estuvieron entre los }\end{array}$ & rojos & $\begin{array}{l}\text { negué sé yo, entre los } \\
\text { negros o entre los } \\
\text { falangistas }\end{array}$ \\
\hline 29 & $19-\mathrm{F}$ & Rayuela & $\begin{array}{l}\text { son de tal raza, todos } \\
\text { los habitantes de piel }\end{array}$ & roja & $\begin{array}{l}\text { tales una gran parte } \\
\text { de etíopes de piel } \\
\text { rojiza oscura, }\end{array}$ \\
\hline 30 & $19-\mathrm{F}$ & $\begin{array}{l}\text { Sobre } \\
\text { héroes y } \\
\text { tumbas }\end{array}$ & $\begin{array}{l}\text { usted, le he tomado } \\
\text { cariño. } \\
\text { Se ha puesto usted }\end{array}$ & rojo & $\begin{array}{c}\text { Perdone } \\
\text { debía haber dicho. - - } \\
\text { No }\end{array}$ \\
\hline 31 & $19-N$ & España: ABC & $\begin{array}{c}\text { poco antes de que } \\
\text { alcanzase la órbita del } \\
\text { Planeta }\end{array}$ & Rojo & $\begin{array}{l}\text { Varias teorías han } \\
\text { intentado explicar este } \\
\text { nuevo fracaso de la }\end{array}$ \\
\hline 32 & $19-\mathrm{N}$ & $\begin{array}{l}\text { Méx: } \\
\text { Yucatán: } \\
\text { 97Jun18 }\end{array}$ & $\begin{array}{c}\text { los gritos desesperados } \\
\text { del entrenador Maldini } \\
\text { que exigía la }\end{array}$ & roja & $\begin{array}{l}\ldots \text { no obstante los } \\
\text { aficionados rechiflan al } \\
\text { mediocampista }\end{array}$ \\
\hline 33 & $19-\mathrm{F}$ & la & $\begin{array}{l}\text { Oriental en su gran } \\
\text { mayoría de ellos, etc. } \\
\text { Raza }\end{array}$ & Roja & $\begin{array}{c}\text { son de tal raza, } \\
\text { todos los habitantes de } \\
\text { piel roja }\end{array}$ \\
\hline
\end{tabular}

\subsubsection{Análisis de las coocurrencias}

Durante la segunda fase de nuestra investigación intentaremos analizar cuáles son las coocurrencias más comunes del color rojo. Para ello, es importante definir el concepto de colocación y relacionarlo con las unidades fraseológicas. Coseriu (1981:128) define las "colocaciones» o "clichés léxicos» como sintagmas léxicos prefijados de forma tradicional, y para Gloria Corpas (2003:69) las «colocaciones» son a su vez unidades fraseológicas porque son poliléxicas, sus elementos integrantes coaparecen o coocurren con alta frecuencia y tienen otras características compartidas con los fraseologismos, como la institucionalización, la estabilidad, la idiomaticidad y la posibilidad de variación.

Por este motivo, es importante analizar cuáles son las colocaciones más comunes de este término cromático, ya que nos permitirá ver con qué suele coocurrir el color rojo y si la connotación de este conjunto léxico es mayormente positivo o negativo. Esta diferenciación responde al paradigma que Morley y Partington ofrecieron en su estudio de 2009, donde las valoraciones se ofrecen en realidades opuestas: positivo/negativo, bueno/malo, deseado/no deseado, favorable/no 
favorable, recomendable/no recomendable. A continuación mostramos las 100 colocaciones más frecuentes del término «rojo»:

\begin{tabular}{|c|c|c|}
\hline 1 & cruz & 360 \\
\hline 2 & sangre & 192 \\
\hline 3 & color & 175 \\
\hline 4 & mar & 137 \\
\hline 5 & blanco & 126 \\
\hline 6 & azul & 111 \\
\hline 7 & labios & 98 \\
\hline 8 & verde & 97 \\
\hline 9 & luz & 93 \\
\hline 10 & blanca & 75 \\
\hline 11 & negro & 74 \\
\hline 12 & amarillo & 72 \\
\hline 13 & azules & 71 \\
\hline 14 & lope & 66 \\
\hline 15 & blancas & 63 \\
\hline 16 & francisco & 63 \\
\hline 17 & glóbulos & 61 \\
\hline 18 & verdes & 61 \\
\hline 19 & ejército & 61 \\
\hline 20 & villa & 61 \\
\hline 21 & flores & 52 \\
\hline 22 & alvar & 48 \\
\hline 23 & bandera & 47 \\
\hline 24 & soto & 44 \\
\hline 25 & gabriel & 44 \\
\hline 26 & rojo & 43 \\
\hline 27 & blancos & 43 \\
\hline 28 & enrique & 40 \\
\hline 29 & rosa & 38 \\
\hline 30 & roberto & 37 \\
\hline 31 & terciopelo & 36 \\
\hline 32 & medias & 36 \\
\hline 33 & pelo & 36 \\
\hline 34 & rayos & 34 \\
\hline 35 & sánchez & 33 \\
\hline 36 & luces & 33 \\
\hline 37 & rosas & 32 \\
\hline 38 & vestido & 32 \\
\hline 39 & vivo & 32 \\
\hline 40 & corresponsalía & 31 \\
\hline 41 & alerta & 30 \\
\hline 42 & púrpura & 29 \\
\hline 43 & amarillas & 28 \\
\hline 44 & seda & 28 \\
\hline 45 & oriente & 28 \\
\hline 46 & sindical & 27 \\
\hline 47 & colores & 27 \\
\hline 48 & agustín & 26 \\
\hline 49 & piel & 26 \\
\hline 50 & amarillos & 25 \\
\hline
\end{tabular}

\begin{tabular}{|c|c|c|}
\hline 51 & canciller & 25 \\
\hline 52 & brillante & 25 \\
\hline 53 & oscuro & 25 \\
\hline 54 & señoras & 24 \\
\hline 55 & negros & 24 \\
\hline 56 & clavel & 23 \\
\hline 57 & sandoval & 23 \\
\hline 58 & mejillas & 23 \\
\hline 59 & cuello & 23 \\
\hline 60 & manuel & 23 \\
\hline 61 & intenso & 22 \\
\hline 62 & banda & 22 \\
\hline 63 & vergüenza & 22 \\
\hline 64 & cabellos & 22 \\
\hline 65 & gris & 21 \\
\hline 66 & tinta & 21 \\
\hline 67 & mancha & 21 \\
\hline 68 & negra & 21 \\
\hline 69 & trapo & 20 \\
\hline 70 & manchas & 20 \\
\hline 71 & apolo & 20 \\
\hline 72 & punta & 20 \\
\hline 73 & arçobispo & 19 \\
\hline 74 & claveles & 18 \\
\hline 75 & vestida & 18 \\
\hline 76 & barba & 18 \\
\hline 77 & fernández & 18 \\
\hline 78 & capa & 18 \\
\hline 79 & humor & 18 \\
\hline 80 & zorrilla & 17 \\
\hline 81 & independiente & 17 \\
\hline 82 & tarjeta & 16 \\
\hline 83 & alfombra & 16 \\
\hline 84 & amarilla & 16 \\
\hline 85 & cinta & 16 \\
\hline 86 & roja & 16 \\
\hline 87 & jorge & 16 \\
\hline 88 & pinilla & 15 \\
\hline 89 & algas & 15 \\
\hline 90 & lápiz & 15 \\
\hline 91 & violeta & 15 \\
\hline 92 & grana & 15 \\
\hline 93 & pieles & 15 \\
\hline 94 & guardias & 15 \\
\hline 95 & gallo & 15 \\
\hline 96 & simón & 15 \\
\hline 97 & cristóbal & 15 \\
\hline 98 & manto & 15 \\
\hline 99 & enorme & 15 \\
\hline 100 & nubes & 15 \\
\hline
\end{tabular}

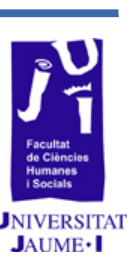


En el presente apartado analizaremos cuál ha sido el número exacto de expresiones metafóricas o en sentido figurado encontradas entre las 700 que hemos analizado, clasificaremos estos resultados y a través de estos analizaremos la prosodia semántica que analizamos durante la segunda fase. El proceso de cuantificación de las expresiones no ha sido difícil debido a que el número de frases metafóricas que hemos encontrado en el corpus se ha mantenido siempre en una cifra manejable. Consideramos que de haber analizado otro color, como el blanco o el negro, habríamos obtenido un mayor número de resultados y también un mayor número de expresiones representativas del sentido figurado.

\subsection{Resultados cuantitativos}

A continuación, ofrecemos la lista de expresiones en sentido figurado en las que aparece el color rojo, teniendo en cuenta el número de apariciones obtenidas en el Corpus del Español de Mark Davies:

\begin{tabular}{|c|c|c|c|c|}
\hline Forma & Palabras & Metafóricas & Porcent. & Ejemplos \\
\hline [rojo] & 7089 & 33 & $0,46 \%$ & \\
\hline rojo & 2372 & 12 & $0,17 \%$ & $\begin{array}{l}\text { - } \text { rojo de la ira } \\
\text { - al rojo vivo (x2) } \\
\text { - alerta rojo } \\
\text { - ponerse rojo } \\
\text { - ponerse rojo como la grana } \\
\text { - rojo como la lumbre } \\
\text { - rojo de cólera } \\
\text { - ponerse rojo de la indignación } \\
\text { - Ejército Rojo (x2) } \\
\text { - Planeta Rojo }\end{array}$ \\
\hline roja & 1741 & 12 & $0,17 \%$ & $\begin{array}{l}\text { - } \text { carne roja }(\times 2) \\
\text { - } \text { tarjeta roja }(\times 3) \\
\text { - } \text { roja como un tomate } \\
\text { - } \text { bandera roja } \\
\text { - } \text { alerta roja }(\times 2) \\
\text { - } \text { piel roja }(\times 2) \\
\text { - } \text { raza roja }\end{array}$ \\
\hline rojos & 806 & 5 & $0,07 \%$ & $\begin{array}{l}\text { - guardias rojos } \\
\text { - rojos como tomates } \\
\text { - los rojos (x3) }\end{array}$ \\
\hline rojas & 2170 & 4 & $0,06 \%$ & $\begin{array}{l}\text { - } \text { volverse rojas [las mejillas] por } \\
\text { la ira } \\
\text { - } \text { pieles rojas }(\mathrm{x} 2) \\
\text { - } \text { tarjetas rojas }\end{array}$ \\
\hline
\end{tabular}




\subsection{Resultados cualitativos}

Una vez recogidos los resultados de nuestro pequeño análisis del sentido figurado de las expresiones con el término "rojo» hemos definido las expresiones para clarificar su significado uno a uno:

\begin{tabular}{|c|c|}
\hline KWIC 1 & $\begin{array}{c}\text { «Estar rojo de ira» es una expresión metafórica que denota un gran } \\
\text { enfado o irritación por algo. }\end{array}$ \\
\hline KWIC 2 & $\begin{array}{l}\text { Los «Guardias rojos» eran un movimiento de masas comunista } \\
\text { promovido en China por Mao Zedong. }\end{array}$ \\
\hline KWIC 3 & $\begin{array}{c}\text { Con «carne roja» se hace referencia a la carne que tiene un color rosado } \\
\text { cuando está cruda. }\end{array}$ \\
\hline KWIC 4 & $\begin{array}{l}\text { Algo que se encuentra «al rojo vivo» tiene la apariencia de brasas } \\
\text { encendidas, ya sea por su color o por la temperatura que ha alcanzado }\end{array}$ \\
\hline KWIC 5 & $\begin{array}{c}\text { "Alerta rojo» (o roja) hace referencia al nivel más alto de amenaza frente } \\
\text { a algo. }\end{array}$ \\
\hline KWIC 6 & Definición KWIC 1. \\
\hline KWIC 7 & $\begin{array}{l}\text { La «tarjeta roja» es la penalización máxima que se puede obtener en el } \\
\text { ámbito deportivo. En ocasiones se utiliza en otros contextos para denotar } \\
\text { algo que no se puede admitir. }\end{array}$ \\
\hline KWIC 8 & $\begin{array}{c}\text { «Ponerse rojo como la grana» implica que las mejillas de una persona } \\
\text { también se vuelven coloradas debido a la vergüenza, otro de los estados } \\
\text { de ánimo que denota el color rojo. }\end{array}$ \\
\hline KWIC 9 & $\begin{array}{l}\text { «Estar rojo como la lumbre» hace referencia a algo que tiene un tono rojo } \\
\text { muy intenso. }\end{array}$ \\
\hline KWIC 10 & $\begin{array}{l}\text { «Estar rojo como un tomate» es una expresión que se utiliza muy a } \\
\text { menudo en español pero que no denota un estado de ánimo concreto. Se } \\
\text { puede «estar rojo como un tomate» debido a un sofoco por hacer un } \\
\text { esfuerzo, al sol, a un enfado puntual, etc. }\end{array}$ \\
\hline KWIC 11 & Definición KWIC 10. \\
\hline KWIC 12 & $\begin{array}{l}\text { Aunque algunos consideran «pieles rojas» como un término ofensivo, se } \\
\text { trata de la forma de referirse a los pueblos indígenas de América y a la } \\
\text { raza a la que pertenecen. }\end{array}$ \\
\hline KWIC 13 & «Estar rojo de cólera» es sinónimo de «estar rojo de ira». \\
\hline KWIC 14 & $\begin{array}{l}\text { «Ponerse rojo de la indignación» implica ponerse rojo también por este } \\
\text { motivo también. }\end{array}$ \\
\hline KWIC 15 & $\begin{array}{c}\text { «Bandera roja» es la bandera socialista y comunista, por lo que enarbolar } \\
\text { la bandera roja puede significar el luchar por estos ideales. }\end{array}$ \\
\hline KWIC 16 & «Los rojos» son los opositores de la derecha, con ideología comunista. \\
\hline KWIC 17 & $\begin{array}{l}\text { El «Ejército Rojo» es el Ejército de la República Socialista Federativa } \\
\text { Soviética de Rusia. }\end{array}$ \\
\hline KWIC 18 & Definición KWIC 17. \\
\hline KWIC 19 & Definición KWIC 5. \\
\hline KWIC 20 & Definición KWIC 7. \\
\hline KWIC 21 & Definición KWIC 16. \\
\hline KWIC 22 & Definición KWIC 12. \\
\hline KWIC 23 & Definición KWIC 4. \\
\hline KWIC 24 & Definición KWIC 5. \\
\hline KWIC 25 & Definición KWIC 3. \\
\hline KWIC 26 & Definición KWIC 12. \\
\hline KWIC 27 & Definición KWIC 7. \\
\hline KWIC 28 & Definición KWIC 16. \\
\hline
\end{tabular}




\begin{tabular}{|c|c|}
\hline KWIC 29 & Definición KWIC 12. \\
\hline KWIC 30 & Definición KWIC 8. \\
\hline KWIC 31 & «Planeta rojo»: se trata de Marte, expresión que se utiliza debido al color \\
& de su superficie terrestre. \\
\hline KWIC 32 & Definición KWIC 7. \\
\hline KWIC 33 & «Raza roja»: Es la raza que poseían los pieles rojas, indígenas de América. \\
\hline
\end{tabular}

\subsubsection{Clasificación de las expresiones con sentido figurado}

De las definiciones anteriores se podría realizar la siguiente clasificación no exhaustiva de la primera fase:

- Grupo 1: estados de ánimo:

- ira, enfado, cólera (volverse rojo de ira/cólera)

○ vergüenza (ponerse rojo de vergüenza, volverse rojo como la grana)

○ indignación (ponerse rojo de indignación)

- Grupo 2: momentos históricos/políticos:

- bandera roja

- los rojos, Ejército Rojo

- pieles rojas/raza roja

○ zona roja

- Grupo 3: indicación de peligro o falta:

○ tarjeta roja

- alerta roja

- números rojos

- Grupo 4: Otros:

o carne roja

- planeta rojo

- marea roja

Como podemos comprobar en la clasificación anterior, la gran mayoría de expresiones que hemos encontrado recogen, además de las diversas connotaciones que el color tiene histórica y políticamente, una connotación negativa (enfado, indignación, vergüenza, etc.), o bien pretenden transmitir una sensación de peligro (alerta, zona roja, etc.). Esto lo confirman, además, expresiones como "línea roja», "luz roja», "teléfono rojo», etc. Tal y como explican Soriano y Valenzuela (2009), la connotación de un conjunto léxico, como los que aparecen en la clasificación anterior, dependen en gran medida de la asociación de los términos cromáticos con situaciones u objetos de la vida real donde este color está presente, como por ejemplo la luz roja que se enciende a menudo en situación de alerta o el color rojo que tiene la sangre. Esto hace que se hayan creado una serie de asociaciones que han llevado a la connotación de algunos términos del color. Si bien esta connotación depende de la experiencia personal de cada uno, puede haber diferencias entre lenguas y colores. 


\subsubsection{Análisis de la connotación}

Durante esta segunda fase, procederemos a analizar los rastros de prosodia semántica obtenidos, teniendo en cuenta aquello que hemos explicado sobre connotación en el marco teórico y valorando la lista de colocaciones que ofrecemos en la fase de análisis de las coocurrencias. Consideramos que es interesante analizar la connotación de las diversas unidades fraseológicas porque este tipo de expresiones dan mucha información sobre la forma de pensar y argumentar en un texto, así que es interesante ver si estas valoraciones son positivas o negativas y esto es un aspecto que se puede estudiar analizando la prosodia semántica presente en estas.

Tomando la lista de coocurrencias más comunes del término cromático "rojo», que hemos incluido en el apartado 4.3.2, hemos realizado la búsqueda de cada unidad léxica $y$, analizando los 100 primeros resultados obtenidos en el $\mathrm{CdE}$, hemos seleccionado aquellas colocaciones que sí que son interesantes para nuestro estudio por presentar sentido metafórico o metonímico. Hemos limitado la búsqueda a cuatro palabras a izquierda y derecha, ya que «rojo» funcionará prácticamente siempre en nuestro caso como adjetivo y no creemos que sea necesario ampliar el rango de búsqueda en este caso.

En un gran número de casos, las coocurrencias no eran colocaciones de ningún tipo, como es el caso del término rojo cuando aparecía con otros términos cromáticos (verde, blanco, azul, etc.), con nombres propios (arçobispo Rojas, Lope Alvar de Rojas, Francisco Rojas, Jesús Villa Rojo, Pedro Soto de Rojas, etc.) o cuando simplemente el término cromático funcionaba como adjetivo calificativo de un sustantivo determinado (labios rojos, flores rojas, rosas rojas, clavel rojo, mejillas rojas, etc.). De la lista, hemos descartado todos estos casos para eliminar el mayor ruido posible a nuestro estudio y también hemos suprimido aquellas expresiones que presentaban significado metonímico, ya que son expresiones en las que simplemente se resalta un aspecto de la realidad (en este caso que la entidad es de color rojo o presenta este color como uno de los que contiene) para hacer referencia al conjunto completo y formar parte de su denominación. Serían los casos de "mar Rojo», "glóbulos rojos», "Medias Rojas», "rayos rojos», "cabellos rojos», "Gran Mancha Roja» y "algas rojas». En la siguiente tabla presentamos aquellas unidades léxicas con sentido figurado que estudiaremos en profundidad. En la segunda columna detallamos cuál es la palabra que coocurre con el término cromático, en la tercera especificamos el número de colocaciones que presenta el CdE para cada caso y en la tercera columna damos la coocurrencia que aparece de forma más común: 


\begin{tabular}{|c|c|c|c|}
\cline { 2 - 4 } \multicolumn{1}{c|}{} & $\begin{array}{c}\text { Palabra } \\
\text { coocurrente }\end{array}$ & $\begin{array}{c}\text { Número de } \\
\text { resultados }\end{array}$ & Colocación más común \\
\hline 9 & luz & 93 & luz roja \\
\hline 19 & ejército & 61 & Ejército Rojo \\
\hline 23 & bandera & 47 & bandera roja \\
\hline 39 & vivo & 32 & al rojo vivo \\
\hline 41 & alerta & 30 & plerta roja \\
\hline 49 & piel & 26 & rojo de vergüenza \\
\hline 63 & vergüenza & 22 & tarjeta roja \\
\hline 82 & tarjeta & 16 & rojo como la grana \\
\hline 92 & grana & 15 & Guardias Rojos \\
\hline 94 & guardias & 15 &
\end{tabular}

Analizar la prosodia semántica de unidades léxicas con términos cromáticos será una tarea difícil ya que los términos cromáticos suelen aparecer como adjetivos calificativos y la carga semántica de estos no es tan valorativa como podrían serlo adjetivos como "bueno», "agradable» o «denigrante». Aún así procederemos a guiarnos por el contexto que nos da cada KWIC y dividiremos el análisis en tres grupos, siguiendo la clasificación no exhaustiva ofrecida en la fase anterior y dejando de lado el grupo 4, que presentaba mayormente unidades léxicas metonímicas como las descartadas anteriormente.

Grupo 1: estados de ánimo

$$
\text { «rojo de vergüenza» }
$$

Prosodia semántica: negativa, vergüenza, ira

Frecuencia: 12/22

(1). [...] La primera clase fue caótica y la vergüenza tiñó de rojo hasta las paredes. Mi madre dijo que, evidentemente, la profesora no servía [...]

(2). [...] Saltamos de la cama, dichosos, aturdidos, y la tía Julia, roja de vergüenza, se acomodaba la ropa y yo, cerrando los ojos, como [...]

(3). [...] estaba muerta», fue lo primero que contra toda evidencia dijo y se puso rojo de vergüenza porque aquello no era más que una disculpa dirigida a calmar el odio [...]

(4). [...] ilos sexos! (todavía, al recordar ese detalle, se ponía roja de vergüenza), y las figuras alrededor de [...]

(5). [...] al salir de la escuela, con lo cual nos poníamos rojos de vergüenza y de ira. Inmediatamente echaba sobre el mostrador una pieza de [...]

(6). [...] el rostro de la pecadora, rojo de vergüenza por primera vez en su vida, ocultóse en el casto pecho de [...]

(7). [...] sillón en un rincón: cayó en él, y abrumó de nuevo la vergüenza roja de su faz entre los brazos... [...]

(8). [...] le pregunté si ya no la quería. Su contestación fue callarse y ponerse rojo de vergüenza $[. .$.

(9). [...] de ser su víctima. Sólo de pensarlo la cara se me pone roja de vergüenza. 
Pero líbreme Dios también de [...]

(10). [...] cavilaciones que le herían y emponzoñaban el alma y sacaban a su rostro el color rojo de la vergüenza. ¿Qué héroe de tan ruin condición era él cuando [...]

(11). [...] Ven acá, quítame esto que me oprime como unas tenazas. Ana estaba roja de vergüenza y de ira. Sentía una indignación tan grande como la cólera de $[\ldots]$

(12). [...] iY allí fue el apuro de la infeliz! Vaciló unos momentos, roja de vergüenza y temblándole la mano; [...]

«rojo como la grana»

Prosodia semántica: negativa, pánico

Frecuencia: $6 / 15$

(1). [...] Ya se ponía pálido como la muerte, ya rojo como la grana. Ora sentía un desvanecimiento parecido al vértigo; [...]

(2). [...] aproximándose luego a ella con inquietud al ver que, después de haberse puesto roja como la grana de su saya, palidecía y vacilaba como si fuera a caerse [...]

(3). [...] D. Faustino se puso rojo como la grana y bajó los ojos al suelo, guardando silencio. [...]

(4). [...] Doña Luz se puso roja como la grana. Toda la sangre de su cuerpo se diría que se le subió a la cabeza. [...]

(5). [...] se había propuesto inaugurar la entrevista, y se presentó también trémulo, también rojo como la grana, prolongando tanto sus mudas reverencias que dieron tiempo a la favorita [...]

(6). [...] Stein se puso rojo como la grana, menos de indignación que de vergüenza. - Sobre que ese [...]

Grupo 2: momentos históricos/políticos:

\author{
«Ejército Rojo» \\ Prosodia semántica: negativa, guerra \\ Frecuencia: 19/61
}

(1). [...] Le conocí en la guerra. Él era capitán del ejército rojo; yo estaba al mando de una compañía de nuestro Glorioso Ejército Nacional. [...]

(2). [...] sobrevivió a los progromos de sus años infantiles y a la barbarie del Ejército Rojo y de los nazis y por eso, [...]

(3). [...] esconde todavía uno de los más fabulosos tesoros artísticos, producto del pillaje del Ejército Rojo, vencedor de la Alemania nazi en la II Guerra Mundial. Cientos de obras [...]

(4). [...] donde dio a conocer las primeras noticias sobre los fabulosos tesoros que el Ejército Rojo escondía en la URSS. [...]

(5). [...] que redujo en una cuarta parte las fuerzas del Ejército Rojo (nombre genérico que servía para denominar a las tropas comunistas desde la Larga Marcha) [...]

(6). [...] Así comenzó la II Guerra Mundial. Dieciséis días más tarde el Ejército Rojo cruzaba la frontera polaca, ocupaba la parte oriental de Polonia y comenzaba la 
sovietización $[\ldots]$

(7). [...] soviéticas por sus respectivos territorios. Sin esperar respuesta a tales exigencias, el Ejército Rojo ocupó dichos estados. La URSS impuso gobiernos propicios y reprimió a todos los elementos [...]

(8). [...] donde fueron hostigadas por la milicia campesina y aniquiladas poco a poco por el Ejército Rojo. Sin embargo, [...]

(9). [...] Gracias a la labor del Ejército Rojo, creado y organizado por Liev Trotski, el régimen soviético salió triunfante de este [...]

(10). [...] el Ejército Rojo soviético atacó la frontera. Prácticamente toda Polonia había sido invadida el 20 de septiembre [...]

(11). [...] mediante una reacción violenta y autodestructiva. En Alemania, la llamada Fracción del Ejército Rojo, más conocida como grupo Baader - Meinhof, efectuó numerosos atracos a bancos y [...]

(12). [...] pero fueron repelidos y derrotados en noviembre por el Ejército Rojo. Wrangel y sus tropas abandonaron Rusia [...]

(13). [...] medida incluida en el comunismo de guerra. A mediados de 1921, el Ejército Rojo había sometido a casi todas las bandas [...]

(14). [...] campo. En esta misma época, el Ejército Rojo invadió Transcaucasia, que volvió a quedar bajo control bolchevique. [...]

(15). [...] cuando el líder ruso lósiv Stalin, buscando la seguridad soviética, utilizó al Ejército Rojo para controlar gran parte de [...]

(16). [...] bombardeó Helsinki y otras ciudades, la armada atacó varios puertos fineses y el Ejército Rojo penetró en el país. Los soviéticos desplegaron veinte divisiones completas a lo largo de [...]

(17). [...] El Ejército Rojo lanzó su primera ofensiva sobre una serie de fortificaciones denominada Línea Mannerheim, [...]

(18). [...] de la Línea Mannerheim, que fue ocupada el 11 de marzo por el Ejército Rojo. La lucha cesó el 12 de marzo de 1940. [...]

(19). [...] y el 13 de febrero Budapest cayó en manos del Ejército Rojo soviético. EI gobierno provisional instituyó, [...]

$$
\text { «bandera roja» }
$$

Prosodia semántica: negativa, movimiento revolucionario

Frecuencia: 3/47

(1). [...] También extraño la bandera roja, que era la de los socialistas liberales rusos, y también la de los bolcheviques. [...]

(2). [...] las muy escasas vacaciones que tomaba, mientras paseaba por los muelles descubrió la bandera roja de la URSS en la popa [...]

(3). [...] y del 48, donde todavía se descarga el palo en los manifestantes de bandera roja y se disuelve a tiros las aglomeraciones de huelguistas, nos dice muy bien que $[\ldots]$

$$
\text { «piel roja» }
$$

Prosodia semántica: positiva (¿’), raza

Frecuencia: 6/26

(1). [...] irrefutablemente la estructura humana, sea la de un chino o la de un piel 
roja. Lenguaje quiere decir residencia [...]

(2). [...] Raza Roja: son de tal raza, todos los habitantes de piel roja, tales una gran parte de etíopes de piel rojiza oscura, donde [...]

(3). [...] cuenta en el Libro que «aquel que sentía la necesidad de ser un piel roja, un guerrero de una época anterior a la leyenda [...]

(4). [...] de civilización. Así la raza negra, la amarilla y la raza de piel roja, cuyos individuos se llamaron atlantes y se esparcieron por el mundo cuando la Atlántida

(5). [...] Planeta, nada más natural que la supresión del negro en África, del piel roja en Estados Unidos, del tagalo en Filipinas, del indio en el Perú. [...]

(6). [...] jefes blancos pasaron Missouri arriba, diciendo que eran amigos de los hombres de piel roja. Este país pertenece a los pieles rojas, pero sus hermanos blancos lo atraviesan [...]

$$
\text { «Guardias Rojos» }
$$

Prosodia semántica: negativa, movimiento revolucionario

Frecuencia: $8 / 15$

(1). [...] socialismo completo, Mao contraatacó y movilizó a la juventud a través de los guardias rojos durante la Revolución Cultural proletaria (1966 - 1969), promovidos para atacar [...]

(2). [...] los enfrentamientos entre maoístas y antimaoístas, así como entre diferentes facciones de los guardias rojos, costaron miles de vidas. En algunas zonas la rebelión condujo a la anarquía [...]

(3). [...] había estado a punto de conquistar en 1849 y que fue ocupado por los guardias rojos en 1966; no obstante, [...]

(4). [...] quiso proporcionarles la experiencia de una auténtica lucha revolucionaria. III. Los Guardias Rojos Y El Auge Revolucionario Mao, además, deseó recuperar su liderazgo, $[\ldots]$

(5). [...] La educación en escuelas y colegios fue suspendida cuando los guardias rojos arremetieron contra la autoridad de maestros, educadores y, en ocasiones, de los $[\ldots]$

(6). [...] Millones de guardias rojos comenzaron a ocupar trenes para viajar por todo el país con el fin de difundir [...]

(7). [...] Los opositores a Mao intentaron tomar la iniciativa formando sus propios grupos de guardias rojos, y se produjeron frecuentes enfrentamientos callejeros.

(8). [...] originar ataques sobre sus culturas y sus identidades, normalmente a cargo de los guardias rojos. La mayor parte del patrimonio histórico nacional resultó dañado o destruido y [...]

Grupo 3: indicación de peligro o falta

$$
\text { «luz roja» }
$$

Prosodia semántica: negativa, amenaza

Frecuencia: 8/92

(1). [...] nos prendió la luz roja a varias personas que nos opusimos a su candidatura, entre estas personas estaba [...] 
(2). [...] Ileno, se da el aviso al maquinista con un timbre o con una luz roja, pero antes de la electricidad, tocaban una campana [...]

(3). [...] tiras cómicas, me veo en el bar de la calle sexta. Una luz roja me señala con vergüenza, me siento hurgando en la vida íntima de otros seres [...]

(4). [...] Hubo de parar un momento. La luz roja lo ordenaba. Y al sentir el remolino bramador e interminable, $[\ldots]$

(5). [...] con sólo una mano izquierda y un rápido reviramiento de cuerpo ágil encendía la luz roja como un aviso de alarma sobre las pobrezas de la verbena llena de humanos semejantes [...]

(6). [...] Un malestar general indicaba que se encendía la luz roja de una caldera apunto de estallar. La playa formaba parte de [...]

(7). [...] la presentó esa noche, hacía ya como seis meses, bajo la tumescente luz roja del Atzimba. «Ésta es Brasil», le dijo [...]

(8). [...] La escena se alumbrará toda con la luz roja de las llamas. Lisardo contempla un momento aterrado tan espantosa visión, [...]

«al rojo vivo»

Prosodia semántica: negativa, amenaza

Frecuencia: $9 / 32$

(1). [...] en una cara redonda y que sufría de una acusada tendencia a ponerse al rojo vivo, a la congestión sanguínea. La miró, Olga, [...]

(2). [...] Antes - recuerdo con cierta sorna - solían mostrarme el rojo vivo de mi propia sangre, pero ese color es ya un hastío. [...]

(3). [...] el diputado ucedeísta Luis Fernando Herrera puso la cuestión al rojo vivo cuando sostuvo que Alsogaray tiene el defecto de creer que el liberalismo [...]

(4). [...] de la crisis El Kremlin admitió ayer que la situación en el país está al rojo vivo, lo cual obligó al presidente de Rusia, [...]

(5). [...] cardenista, eran lucha de rudos, y las pasiones subían a veces al color rojo vivo de sangre. Desfilaban las clientelas [...]

(6). [...] lo aplicó en tres donde los ánimos estaban al rojo vivo. Fue un fracaso. En uno de ellos, Querétaro, se impusieron [...]

(7). [...] cambios de Camerún, sale Ipoua y entra Job, el ambiente se pone al rojo vivo. Miles de aficionados corean «Camerún...camerún», un grito que apaga [...]

(8). [...] Al rojo vivo debate sobre MIDEM y oposición a negocios con Cuba ¿Hasta dónde debe el $[\ldots]$

(9). [...] Escándalo sexual de Clinton al rojo vivo Washington - - El presidente Bill Clinton se vio el viernes más enredado aún [...]

«alerta roja»

Prosodia semántica: negativa, amenaza

Frecuencia: $14 / 30$

(1). [...] mucho más difícil todavía estar encasquetado en un uniforme de bombero y estar en alerta roja todo el tiempo, los hechos transcienden, superan los sucesos de prevención [...]

(2). [...] que aunque no esté en plena actividad beligerante está muchas veces poniéndose en alerta roja, altera la situación del estado y del país, [...] 
(3). [...] se recibió a bordo un alerta rojo. El ataque del guardacostas por parte de aviones británicos fue casi instantáneo. [...]

(4). [...] De inmediato, la Comisión Nacional de Emergencia (CNE) decretó una alerta roja y dispuso varias medidas de seguridad: * Cierre del tránsito por la carretera [...]

(5). [...] doblete de particulares * No es el primero * Prostituyen la función pública

* Alerta roja por incendios en Petén [...]

(6). [...] El Gobierno declaró ayer una alerta roja nacional a causa de la gravedad de los incendios forestales que arrasan gran parte [...]

(7). [...] la Coordinadora Nacional para la Reducción de Desastres declararon alerta roja y evacuaron a unas 250 personas de [...]

(8). [...] quien declaró, en ausencia del mandatario, la emergencia nacional y luego la alerta roja por el fuego. Por ello, [...]

(9). [...] Declaran alerta roja por nueva erupción de volcán Pacaya en Guatemala Guatemala (ACAN-EFE) [...]

(10). [...] Comisión Nacional para la Reducción de Desastres (CONRED) decidió declarar la «alerta roja» en la zona pero todavía no se ha realizado ninguna evacuación, [...]

(11). [...] dijo que se ha decretado una "alerta roja» en la zona debido a la nueva actividad volcánica. [...]

(12). [...] domingo unas 1.500 personas, explicó el funcionario. El gobierno decretó el sábado alerta roja para coordinar acciones de los diversos organismos en la lucha contra los siniestros y [...]

(13). [...] El Comité de Emergencia Nacional (COEN) de El Salvador declaró ayer, martes, "Alerta Roja» debido al elevado número de incendios forestales registrados en este país, [...]

(14). [...] Eddy Sánchez, director del Observatorio Nacional. Se decretó una «alerta roja» para las aldeas próximas al cráter, [...]

«tarjeta roja»

Prosodia semántica: negativa, penalización

Frecuencia: 8/16

(1). [...] Bueno Tomás, tarjeta roja, la entrada ha sido a tu juicio merecedora de amarilla [...]

(2). [...] que había salido de la caseta, le preguntó si era tarjeta amarilla o tarjeta roja y sacó tarjeta roja y - y vamos, le expulsó. Entonces juzgando [...]

(3). [...] sancionado ayer el defensor saprissista Rónald González, quien el domingo anterior vio la tarjeta roja en el primer juego [...]

(4). [...] se originó una situación en la que José Adames fue penalizado con tarjeta roja, hecho que provocó posteriormente la salida del dirigente norteño, José Morel. [...]

(5). [...] de lugar. RAMÓN Ramírez dice algo al árbitro y se va con la tarjeta roja a cuestas... iVaya final no apto para cardiacos! [...]

(6). [...] Nankov, m. 43 Benítez, m. 46 Stoitchkov, m. 72 Ivanov:. m. 87, Tarjeta roja a Nankov por doble amonestación. [...]

(7). [...] Al minuto 47 Darryl Powell recibe su segunda amonestación y por lo tanto 
la tarjeta roja, luego que en dos ocasiones seguidas derriba al propio Ortega. [...]

(8). [...] impedir el saque de una falta. Un árbitro puede mostrar la tarjeta roja a un jugador y expulsarle del terreno de juego [...]

\section{Discusión y conclusiones}

Se confirma que en el análisis de la connotación de estos términos cromáticos, no es suficiente con el paradigma bipolar de Morley y Partington (2009), ya que no hay un solo polo positivo o bueno y uno negativo o malo. En las expresiones del primer grupo y en las del tercer grupo, comprobamos que el polo negativo es más claro. En el primer grupo, el término cromático "rojo» se ha utilizado cuando las personas o entidades que intervenían sufrían una situación de vergüenza, de ira o de palidez físicamente visible en el rostro y, en el tercer grupo, el color rojo se ha empleado en situaciones de alerta, de peligro o amenaza.

Sin embargo, vemos que en el segundo grupo, el significado o la connotación de este color no está tan claro, ya que estas unidades refieren a contextos políticos que, vistos desde un determinado prisma o punto, pueden verse como positivos o negativos (socialismo, revolución, etc.). Consideramos que la prosodia semántica debería ir más allá y analizar realidades a otro nivel, diferenciando además aquellas unidades léxicas que se mantienen neutras o que por el motivo que sea (político, cultural, personal) no se puede valorar de forma objetiva.

Con toda la información extraída, consideramos que el papel semántico de los colores dentro de las unidades fraseológicas es muy interesante, ya que estos ofrecen una valoración (ya sea positiva, negativa, neutra o no claramente valorable) sobre aquello de lo que se está hablando. Este análisis se enmarca en un estudio mayor donde se pretende analizar, de forma comparativa entre el idioma español y el alemán, cuál es el papel que tienen los términos del color dentro de las lenguas y si pueden servir para expresar en un idioma aspectos que en el otro se expresan de forma completamente diferente, por lo que, aunque el estudio del "rojo» nos ha dado mucha información sobre el papel de los términos cromáticos, esperamos poder ir más allá y ampliar la paleta de colores.

\section{Bibliografía}

BAKER, M. et al. (1993): Text and Technology. In Honour of John Sinclair, John Benjamins Publishing Company, Ámsterdam y Filadelfia.

CORPAS PASTOR, G. (2003): Diez años de investigación en fraseología: Análisis sintáctico-semánticos, contrastivos y traductológicos, VervuertIberoamericana, Fráncfort del Meno-Madrid.

COSERIU, E. (1981): Lecciones de lingüística general, Gredos, Madrid. 
Instituto de InVEStigación Rafael LapeSA de la ReAl Academia Española (2013): Corpus del Nuevo diccionario histórico (CDH) [en línea]. <http://web.frl.es/CNDHE> [Consulta: 7 de septiembre de 2009]

LAKOFF, G. y M. JOHnSON (1980): Metáforas de la vida cotidiana, University of Chicago Press, Chicago.

Louw, B. (1993): "Irony in the Text or Insincerity in the Writer? The Diagnostic Potential of Semantic Prosodies", BAKER, M. , et al. (ed.): Text 703 and Technology: In Honour of John Sinclair, Benjamins, Ámsterdam y Filadelfia, p. 240-251.

Morley, J. y A. Partington (2009): «A Few Frequently Asked Questions about Semantic -or Evaluative- Prosody». International Journal of Corpus Linguistics, 14(2), p. 139-158.

Real Academia Española. Diccionario de la Real Academia Española [en línea]. Vigesimotercera edición. <http://lema.rae.es/drae/?val=corpus>. [Consulta: 27 de enero de 2015].

- : Banco de datos (CORDE) [en línea]. Corpus diacrónico del español. <http://www.rae.es>. [Consulta: 7 de septiembre de 2015].

- : Banco de datos (CREA) [en línea]. Corpus de referencia del español actual. <http://www.rae.es>. [Consulta: 7 de septiembre de 2015].

Soriano, C. y J. Valenzuela Manzanares (2009): «Emotion and Colour Across Languages: Implicit Associations in Spanish Colour Terms». Social Science Information, Special Issue: The Language of Emotion-Conceptual and Cultural Issues, p. 421-445.

Steen, G. J. (2007): «Finding Metaphor in Discourse: Pragglejaz and Beyond». Culture Language and Representation, V, p. 9-25.

Tognini-BonelLI, E. (2001): Corpus Linguistics at Work, John Benjamins Publishing Company, Ámsterdam y Filadelfia. 